PROCEEDINGS OF THE

AMERICAN MATHEMATICAL SOCIETY

Volume 133, Number 11, Pages 3235-3241

S 0002-9939(05)07902-5

Article electronically published on June 20, 2005

\title{
A COVERING LEMMA FOR RECTANGLES IN $\mathbb{R}^{n}$
}

\author{
ROBERT FEFFERMAN AND JILL PIPHER \\ (Communicated by Andreas Seeger)
}

\begin{abstract}
We prove a covering lemma for rectangles in $\mathbb{R}^{n}$ which has connections to a problem of Zygmund and its solution in three dimensions by Cordoba.
\end{abstract}

One of the objectives of this note is to revive interest in a collection of problems in differentiation theory related to a conjecture of Zygmund. We shall also give another proof of the sharp mapping properties near $L^{1}$ of the strong maximal operator by proving a covering lemma which is in the spirit of [2].

The classical operators of harmonic analysis which are important in various fields of analysis and PDE include singular integrals, maximal functions, Hilbert and Riesz transforms and other operators defined by multipliers. Some of these operators are invariant under the usual dilation group of $\mathbb{R}^{n}$; others, like the multiple Hilbert transform, are invariant under the product dilations, but there are others which transform in such a way as to be invariant under some other group of dilations.

In $\mathbb{R}^{n}$, the product dilations have the form

$$
\rho_{\delta_{1}, \delta_{2}, \ldots, \delta_{n}}\left(x_{1}, \ldots, x_{n}\right)=\left(\delta_{1} x_{1}, \delta_{2} x_{2}, \ldots, \delta_{n} x_{n}\right) .
$$

The mapping properties of operators invariant under this group of dilations on $L^{p}$ in the range $1<p<\infty$ typically follow from the one-parameter theory via iterative or vector-valued methods. The differences between the product dilation case and the usual dilation case are most apparent in the sharp estimates near $L^{1}$. For example, consider the usual Hardy-Littlewood maximal operator in $\mathbb{R}^{n}$ :

$$
M f(x)=\sup _{x \in Q} \frac{1}{|Q|} \int_{Q} f d x
$$

where the sup is taken over cubes in $\mathbb{R}^{n}$ with sides parallel to the axes. The product dilation version of this operator is the strong maximal operator in $\mathbb{R}^{n}$ :

$$
M_{n} f(x)=\sup _{x \in R} \frac{1}{|R|} \int_{R} f d x
$$

where $R$ is a rectangle with sides parallel to the axes. On the one hand, $M f$ satisfies the familiar weak type inequality near $L^{1}$ :

$$
|\{x: M f(x)>\lambda\}|<C \frac{\|f\|_{L^{1}}}{\lambda},
$$

Received by the editors April 23, 2004

2000 Mathematics Subject Classification. Primary 42B20.

(C)2005 American Mathematical Society 
whereas $M_{n} f$ satisfies

$$
\left|\left\{x: M_{n} f(x)>\lambda\right\}\right|<C \int \frac{f}{\lambda}\left(1+\log ^{+}\left(\frac{f}{\lambda}\right)^{n-1}\right) d x .
$$

The product dilation group however is only one example of a multiparameter dilation group. (For a very general point of view, see [5.) Consider, for example, the dilations $\left(x_{1}, x_{2}, x_{3}\right) \mapsto\left(\delta_{1} x_{1}, \delta_{2} x_{2}, \delta_{1} \delta_{2} x_{3}\right)$ in $\mathbb{R}^{3}$ and the multipliers $m$ which are invariant under these dilations. Then, viewing operators $T_{m}$ associated to these multipliers as three-parameter product operators, one can see that these $T_{m}$ are bounded on $L^{p}\left(\mathbb{R}^{3}\right)$ in the range $1<p<\infty$. This fact is related to the following. If one forms a maximal function $M_{s, t}$ by averaging over rectangles in $\mathbb{R}^{3}$ with sidelengths $s \times t \times s t$, then $M_{s, t}$ is clearly dominated by $M_{3}$, the strong maximal function in $\mathbb{R}^{3}$. However, it turns out that the maximal function $M_{s, t}$ associated to this dilation structure behaves more like $M_{2}$, the two-dimensional strong maximal function. This was demonstrated by A. Córdoba [2] who showed that, for $Q$ the unit cube,

$$
\left|\left\{\left(x_{1}, x_{2}, x_{3}\right) \in Q: M_{s, t} f\left(x_{1}, x_{2}, x_{3}\right)>\lambda\right\}\right| \leq \frac{C}{\lambda}\|f\|_{L \log L(Q)} .
$$

That is, near $L^{1}, M_{s, t}$ and $M_{2}$ have the same mapping properties. (E. Stein has observed that the properties of this type of maximal operator are related to boundary value problems for Poisson integrals on symmetric spaces. In [4], the behavior near $L^{1}$ of singular integral operators associated to multipliers invariant under this dilation group was studied.)

There are several interesting features of this result and its proof. First, the weak type inequality for the maximal function is established by means of a covering lemma. Second, the proof has not been generalized to higher dimensions. There are, of course, many possible higher-dimensional analogs of this results, but even the most straightforward of these remains elusive. Third, the result gave support to a conjecture of Zygmund regarding the concept of a $k$-parameter family of dilation invariant operators in $\mathbb{R}^{n}$. Zygmund had conjectured that if the rectangles in $\mathbb{R}^{n}$ had $n$ side lengths which involved only $k$ independent variables, then the resulting maximal operator should behave like $M_{k}$, the $k$-parameter strong maximal operator. F. Soria showed in [6] that the question required more assumptions than Zygmund had suppposed. However, it seems natural to conjecture that some modified version of Zygmund's conjecture should hold, one that includes, say, the dilation family $\left(\delta_{1}, \ldots, \delta_{n-1}, \delta_{1} \delta_{2} \ldots \delta_{n-1}\right)$. Finally, we note that estimates in Orlicz spaces are more delicate as they cannot be obtained by iteration.

Here is the idea behind Córdoba's result. Consider a finite family of rectangles $\left\{R_{i}\right\}$ in $\mathbb{R}^{3}$, sides parallel to the axes, whose side lengths are of the form $s \times t \times s t$. Order the rectangles according to the size of the third side: $s_{1} t_{1}>s_{2} t_{2}>\ldots>$ $s_{N} t_{N}$. If one can choose a subsequence of these rectangles, denoted $\left\{\tilde{R}_{i}\right\}$, which satisfies:

(i) $\left|\bigcup R_{i}\right| \leq 10\left|\bigcup \tilde{R}_{i}\right|$,

(ii) $\int_{\bigcup \tilde{R}_{i}} \exp \left(\sum_{i} \chi_{\tilde{R}_{i}}\right) d x d y d z \leq C\left|\bigcup \tilde{R}_{i}\right|$, 
then the weak type inequality for $L \log L$ functions holds. To achieve (i) and (ii), Córdoba chooses an $R_{i}$ to be in the subsequence if

$$
\int_{R_{i}} \exp \left(\sum_{k<i} \chi_{\tilde{R}_{k}}\right) d x d y d z \leq c\left|R_{i}\right| .
$$

It is now straightforward to verify that inequality (ii) holds if $c$ is chosen appropriately, but it is trickier to see that the union of rectangles not chosen has small volume.

To see this, let $R_{i}$ be a rectangle not satisfying the selection criterion, i.e., the above inequality fails. From the ordering of the third sides, we see that we can slice away one dimension and that we have

$$
\int_{R_{i}^{\prime}} \exp \left(\sum_{k<i} \chi_{\tilde{R}_{k}^{\prime}}(x, y, z)\right) d x d y \geq c\left|R_{i}^{\prime}\right|
$$

for all $z$ in $R_{i}^{\prime}$, and where $R_{i}^{\prime}=I_{i} \times J_{i}$ is the two-dimensional rectangle of side lengths $s_{i} \times t_{i}$. If $k<i$, the rectangles $R_{k}^{\prime}$ split into two classes - those for which $s_{k}>s_{i}$ and those for which $t_{k}>t_{i}$. This is forced by the condition $s_{k} t_{k}>s_{i} t_{i}$. If one assumes that all the side lengths are dyadic (with no loss of generality), then $s_{k}>s_{i}$ entails the geometric condition that $I_{i} \subset I_{k}$. Let's say $k \in A_{i}$ if $s_{k}>s_{i}$ and that $k \in B_{i}$ if $t_{k}>t_{i}$. Then,

$$
\int_{R_{i}^{\prime}} \exp \left(\sum_{k<i} \chi_{\tilde{R}_{k}^{\prime}}(x, y)\right) d x d y=\int_{R_{i}^{\prime}} \exp \left(\sum_{k \in A_{i}} \chi_{\tilde{R}_{k}^{\prime}}(y)\right) \exp \left(\sum_{k \in B_{i}} \chi_{\tilde{R}_{k}^{\prime}}(x)\right) d x d y,
$$

which splits into a product of two one-dimensional integrals. One can now argue that each $R_{i}$ not chosen must be contained in

$$
\left\{(x, y, z): M_{1}\left(\exp \left(\sum_{k} \chi_{\tilde{R}_{k}^{\prime}}(x, y, z)\right)\right) \times M_{2}\left(\exp \left(\sum_{k} \chi_{\tilde{R}_{k}^{\prime}}(x, y, z)\right)\right)>c^{\prime}\right\}
$$

where $M_{1}$ and $M_{2}$ are one-dimensional maximal operators in the variables $x$ and $y$. By the weak type 1-1 result (1), the set above has measure bounded by a constant times

$$
\int_{\cup R_{i}} \exp \left(\sum_{k} \chi_{\tilde{R}_{k}}\right) d x d y d z .
$$

By the inequality in (ii), we see that the volume of the rectangles discarded by the selection procedure is bounded by $C\left|\cup \tilde{R}_{i}\right|$.

This argument fails in higher dimensions, apparently for two reasons. First, the splitting of the exponential of the sum into a product of one variable (or lower-dimensional) integrals fails. Second, the weak type $L^{1}$ estimate for the onedimensional maximal operator was used at a crucial point to estimate the volume of the discarded rectangles. In higher dimensions, the weak type estimate involves estimates in $L(\log L)^{k}$.

One of the main technical contributions of this note is to show that the second apparent obstacle above can be overcome.

To see this, we shall prove a covering lemma for rectangles in $\mathbb{R}^{n}$, in the spirit of Córdoba's argument, by selecting those rectangles which satisfy the appropriate exponential estimate. The lemma falls short of proving the conjectured weak type estimates for, say, the three-parameter maximal operator defined by the dilation 
structure $s \times t \times u \times s t u$, but it will give new covering lemmas for other families of rectangles.

Let $\left\{R_{i}\right\}$ be an arbitrary family of (dyadic) rectangles in $\mathbb{R}^{n}$ with sides parallel to the axes, and ordered according to decreasing length of one of the sides. To be definite, let $R_{i}=R_{i}^{\prime} \times I_{n}$ and order according to decreasing size of $I_{n}$. Choose a subcollection $\tilde{R}_{i}$ according to the rule:

$$
\int_{R_{i}} \exp \left(\sum_{k<i} \chi_{\tilde{R}_{k}}\right)^{1 /(n-1)} d x_{1} \ldots d x_{n} \leq c\left|R_{i}\right|
$$

Then, we claim that

(i) $\left|\bigcup R_{i}\right| \leq 10\left|\bigcup \tilde{R}_{i}\right|$,

(ii) $\int_{\bigcup \tilde{R}_{i}}\left(\sum_{i} \chi_{\tilde{R}_{i}}\right)^{(n-2) /(n-1)} \exp \left(\sum_{i} \chi_{\tilde{R}_{i}}\right)^{1 /(n-1)} d x_{1} \ldots d x_{n} \leq C\left|\bigcup \tilde{R}_{i}\right|$.

Notice that the estimate in (ii) contains an apparent improvement over what can be deduced automatically from the selection procedure. The exponent in (ii) is sharp - a larger exponent would imply an even better (and false) covering property of the standard family of $n$-parameter rectangles. Then

Assuming condition (ii) holds, it is easy to prove (i). Suppose $R_{i}$ was not chosen.

$$
\int_{R_{i}^{\prime}} \exp \left(\left(\sum_{k<i} \chi_{\tilde{R}_{k}^{\prime}}\right)^{1 /(n-1)}\right) d x_{1} \ldots d x_{n-1}>c\left|R_{i}^{\prime}\right| .
$$

All the rectangles which contribute to this sum have sides $I_{i}$ which must contain $I_{n}$, so we have "sliced away" one dimension in this integral. Thus the collection of rectangles not chosen is contained in the set

$$
\left\{\left(x_{1}, \ldots, x_{n}\right): M_{n-1}\left(\exp \left(\sum_{k} \chi_{\tilde{R}_{k}}(x)\right)^{1 /(n-1)}\right)>c\right\}
$$

Arguing inductively, since $M_{n-1}$ maps $L(\log L)^{n-2}$ into weak $L^{1}$, the measure of this set is bounded by the integral in condition (ii). It remains to prove (ii). Consider the integral

$$
\begin{gathered}
\int_{\cup \tilde{R}_{i}}\left(\sum_{i} \chi_{\tilde{R}_{i}}\right)^{(n-2) /(n-1)} \exp \left(\sum_{i} \chi_{\tilde{R}_{i}}\right)^{1 /(n-1)} \\
=\sum_{p=0}^{\infty} \frac{1}{p !} \int_{\bigcup \tilde{R}_{i}}\left(\sum_{i} \chi_{\tilde{R}_{i}}\right)^{(p+n-2) /(n-1)}
\end{gathered}
$$

For $p=l(n-1)+h$, with $h=1,2, \ldots, n-1$, we have a family of estimates to prove. We first prove it for $p=l(n-1)+1$, and then sketch the argument that the other estimates follow from this. That is, we shall first prove the following inequality:

$$
\sum_{l=0}^{\infty} \frac{1}{(l(n-1)+1) !} \int_{\bigcup \tilde{R}_{i}}\left(\sum_{i} \chi_{\tilde{R}_{i}}\right)^{l+1} \leq C\left|\bigcup \tilde{R}_{i}\right| .
$$


Let $c_{m}(j)$ denote $\int_{R_{m}}\left(\sum_{i<m} \chi_{\tilde{R}_{i}}\right)^{j /(n-1)}$. The selection assumption is equivalent to the condition that $\frac{1}{\left|R_{m}\right|} \sum_{j} \frac{c_{m}(j)}{j !} \leq c$, for all $m$. Fixing $l$ for the moment, consider

$$
\begin{aligned}
& \int_{\bigcup \tilde{R}_{i}}\left(\sum_{i} \chi_{\tilde{R}_{i}}\right)^{l+1} \\
& =\int_{\cup \tilde{R}_{i}} \sum_{(l+1) \text {-tuples }} \chi_{R_{i_{1}}} \chi_{R_{i_{2}}} \chi_{R_{i_{l+1}}} .
\end{aligned}
$$

The sum over all $(l+1)$-tuples of $R_{i}$ 's can be subdivided into a sum over all $(l+1)$ tuples such that $m$ is the largest index and such that $\chi_{R_{m}}$ occurs $k$ times in the sum. There are $C(l+1, k)=\frac{(l+1)(l) \ldots(l+1-k+1)}{k !}$ slots in which $R_{m}$ may occur. Therefore the above sum is bounded by

$$
\begin{aligned}
\int \sum_{m} \sum_{k=1}^{l+1} C(l+1, k) \chi_{R_{m}}^{k} \sum_{\left(i_{1}, \ldots, i_{l+1-k}\right), i<m} \chi_{R_{i_{1}} \ldots \chi_{R_{i_{l+1-k}}}} \\
=\sum_{m} \sum_{k=1}^{l+1} C(l+1, k) \int_{R_{m}}\left(\sum_{i<m} \chi_{R_{i}}\right)^{l+1-k} .
\end{aligned}
$$

Hence, the sum in (7) is bounded by the expression

$$
\begin{aligned}
& \sum_{l} \sum_{m} 1 /(l(n-1)+1) ! \sum_{k=1}^{l+1} C(l+1, k) c_{m}((n-1)(l+1-k)) \\
& \quad \leq \sum_{m} \sum_{k=1}^{\infty} \sum_{l \geq k-1} 1 /(l(n-1)+1) ! C(l+1, k) c_{m}((n-1)(l+1-k)) .
\end{aligned}
$$

Set $q=l+1-k$. The sum above is the same as

$$
\sum_{m} \sum_{q=0}^{\infty} \frac{c_{m}((n-1) q)}{q(n-1) !} \sum_{k=1}^{\infty} \frac{(q(n-1)) !}{((q+k-1)(n-1)+1) !} C(q+k, k) .
$$

This is bounded by a constant times $\sum_{m}\left|R_{m}\right|$ because

$$
\sum_{k=1}^{\infty} \frac{(q(n-1)) !}{((q+k-1)(n-1)+1) !} C(q+k, k) \leq C,
$$

as a straightforward computation verifies. In fact, the $k$ th term in the sum is bounded, when $n \geq 2$, by $1 / k$ !.

We now sketch the argument that it suffices to prove the estimate (ii) for the exponent $p=l(n-1)+1$. Let's just look at the three-dimensional situation for simplicity. Suppose we have shown that

$$
\sum_{p=0}^{\infty} \frac{1}{p !} \int_{\bigcup \tilde{R}_{i}}\left(\sum_{i} \chi_{\tilde{R}_{i}}\right)^{(p+1) / 2} \leq C\left|\bigcup \tilde{R}_{i}\right|,
$$

when $p=(2 k+1)$. 
That same sum, with $p=2 k$, is sandwiched in between two sums with even terms. That is,

$$
\begin{aligned}
& \sum_{k=0}^{\infty} \frac{1}{(2 k) !} \int_{\cup \tilde{R}_{i}}\left(\sum_{i} \chi_{\tilde{R}_{i}}\right)^{(2 k+1) / 2} \\
& \quad \leq \sum_{k=0}^{\infty} \frac{1}{(2 k) !}\left(\int_{\cup \tilde{R}_{i}}\left(\sum_{i} \chi_{\tilde{R}_{i}}\right)^{k}\right)^{1 / 2}\left(\int_{\cup \tilde{R}_{i}}\left(\sum_{i} \chi_{\tilde{R}_{i}}\right)^{k+1}\right)^{1 / 2} .
\end{aligned}
$$

We multiply the $k$-th term integrals above by $1 /(2 k-1)$ ! and $1 /(2 k+1)$ ! respectively, and the result is bounded by

$$
\sum_{k=0}^{\infty} \sqrt{\frac{2 k+1}{2 k}}\left(\frac{1}{(2 k-1) !} \int_{\cup \tilde{R}_{i}}\left(\sum_{i} \chi_{\tilde{R}_{i}}\right)^{k}\right)^{1 / 2}\left(\frac{1}{(2 k+1) !} \int_{\bigcup \tilde{R}_{i}}\left(\sum_{i} \chi_{\tilde{R}_{i}}\right)^{k+1}\right)^{1 / 2},
$$

and Cauchy-Schwarz shows that this is, in turn, bounded by (8). The $n$-parameter situation requires a similar splitting, with different weights, and Hölder's inequality instead of Cauchy-Schwarz.

The arguments above now yield the following general result.

Theorem 0.1. Let $R_{i}$ be a collection of measurable sets in $\mathbb{R}^{n}$. Take $\beta \in(0,1]$ (above we took $\beta=1 /(n-1)$ ). Select a subcollection $\widetilde{R}_{i}$ according to the rule

$$
\int_{R_{m}} \exp \left(\sum_{i<m} \chi_{\widetilde{R}_{i}}(x)\right)^{\beta} d x \leq c\left|R_{m}\right|
$$

Then, one has the improvement

$$
\int\left(\sum_{i} \chi_{\widetilde{R}_{i}}(x)\right)^{1-\beta} \exp \left(\sum_{i} \chi_{\widetilde{R}_{i}}(x)\right)^{\beta} d x \leq C \sum_{m}\left|\widetilde{R}_{m}\right| .
$$

When the $R_{i}$ are intervals in $\mathbb{R}^{n}$, further assumptions in the ordering will also give $\left|\bigcup R_{i}\right| \leq C\left|\bigcup \widetilde{R}_{m}\right|$

The two-dimensional version of this covering lemma has the following stronger feature: it is not necessary to order the rectangles by side length. It suffices to assume that the rectangles do not satisfy any containment relations. The type of 4-dimensional maximal operator to which this particular covering lemma applies includes dilation structures such as $\left(\delta_{1}, \delta_{2}, \delta_{3}, \delta_{1} \delta_{2}\right)$. The remaining obstacle to a true generalization of Zygmund's conjecture is to find the appropriate replacement for the splitting of the two-parameter integral into a product of two one-variable integrals.

Final remarks. It appears that the problem of understanding what makes an $n$ dimensional family of rectangles, and the associated family of SIO's, a $k$-paramenter family is a delicate one. For example, in [6], the following phenomenon is noted. Consider the collection of rectangles whose side lengths are $s \times 1 / s \times t$ for positive $s$, $t$. This is not, strictly speaking, a basis of rectangles. Nevertheless, it is interesting to observe that Córdoba's argument applies to this collection; hence there exists an exponential covering lemma which shows that a maximal operator associated to this collection is really a two-parameter operator. However, the two-dimensional collection of rectangles of the form $s \times 1 / s$ is already a two-parameter family (as examples show). It thus appears that, to this two-parameter family, one may add an independent third side, and yet this does not result in a three-parameter family. 
One way to think about this, and related, phenomena is to try to understand the action of the associated maximal operator on a point mass. When can the geometric nature of the operator be deduced from its action on the delta? This question is closely related to the following issue. Take an $n$-dimensional family of rectangles, like the ones we've been considering. Form the subfamily of rectangles which all have a fixed volume, say volume $=1$. When can the action of the operator be understood in general by its restriction to this subclass? An answer to this question could provide an inductive means of proving higher-dimensional results. For example, for the class $s \times t \times s t$, the subclass is simply rectangles of the form $s \times 1 / s \times 1$, a two-parameter family. And for Soria's class above, the volume $=1$ subclass also has the form $s \times 1 / s \times 1$, which makes it perhaps less surprising that Córdoba's argument applies to this operator as well.

\section{ACKNOWLEDGEMENTS}

The referee made several helpful comments.

\section{REFERENCES}

[1] S.Y. A. Chang and R. Fefferman Some recent developments in Fourier analysis and $H^{p}$ theory on product domains, Bull. A.M.S., vol 12 [1985], 1-43. MR 0766959 (86g:42038)

[2] A. Córdoba, Maximal functions, covering lemmas and Fourier multipliers, Proc. Sympos. Pure Math. vol. 35, American Math. Society, Providence, RI [1979], 29-49. MR0545237 (81f:42017)

[3] A. Córdoba and R. Fefferman, A geometric proof of the strong maximal theorem, Annals of Math., vol 102, [1975], 95-100. MR.0379785 (52:690)

[4] R. Fefferman and J. Pipher, Multiparameter Operators and Sharp Weighted Inequalities, Amer. J. Math., vol 11 (No 2), [1997], 337 - 369. MR.1439553 (98b:42027)

[5] F. Ricci and E. Stein, Mulltiparameter singular integrals and maximal functions, Ann. Inst. Fourier (Grenoble), vol 42, [1992], 637-670. MR.1182643 (94d:42020)

[6] F. Soria, Some examples and counterexamples to a conjecture in the theory of differentiation, Annals of Math.(2), vol 123, [1986], 1-9. MR.0825837|(88a:42026)

Department of Mathematics, University of Chicago, Chicago, Illinois 60637

E-mail address: raf@math.uchicago.edu

Department of Mathematics, Brown University, Providence, Rhode Island 02912

E-mail address: jpipher@math.brown.edu 